\title{
Impact of decentralisation of antiretroviral therapy services on HIV testing and care at a population level in Agago District in rural Northern Uganda: results from the Lablite population surveys
}

\author{
George Abongomera ${ }^{a, b, *}$, Sylvia Kiwuwa-Muyingo ${ }^{c}$, Paul Revill ${ }^{d}$, Levison Chiwaula ${ }^{e}$, Travor Mabuguf, \\ Andrew N. Phillips ${ }^{g}$, Elly Katabira ${ }^{h}$, Adrienne K. Chan ${ }^{\mathrm{e}, \mathrm{i}}$, Charles Gilks $s^{\mathrm{j}, \mathrm{k}}$, Victor Musiime ${ }^{\mathrm{a}, \mathrm{l}}$, James Hakim ${ }^{f}$, \\ Cissy Kityo ${ }^{a}$, Robert Colebunders ${ }^{b}$, Diana M. Gibb ${ }^{m}$, Janet Seeley ${ }^{c, n}$ and Deborah Ford ${ }^{m}$, on behalf of \\ the Lablite Project Team
}

\begin{abstract}
${ }^{a}$ Department of Research, Joint Clinical Research Centre, P.O. Box 10005, Kampala, Uganda; ${ }^{b}$ Faculty of Medicine and Health Sciences, University of Antwerp, 2610 Wilrijk, Antwerp, Belgium; ' Department of Statistics, Medical Research Council/Uganda Virus Research Institute, P.O. Box 49, Entebbe, Uganda; ${ }^{d}$ Centre for Health Economics, University of York, York, YO10 5DD, UK; ${ }^{e}$ Department of Research, Dignitas International, P.O. Box 1071, Zomba, Malawi; ${ }^{f}$ Clinical Research Centre, University of Zimbabwe, P.O. Box MP 167 Harare, Zimbabwe; ${ }^{9}$ Department of Infection \& Population Health, University College London, London, WC1E 6JB, UK; ${ }^{h}$ Department of Research, Infectious Diseases Institute, Makerere University, P.O. Box 22418, Kampala, Uganda; 'Department of Medicine, University of Toronto, Toronto, ON M5S 1A8, Canada; ${ }^{j}$ Faculty of Medicine, Imperial College London, London, SW7 2AZ, UK; ${ }^{~}$ School of Population Health, University of Queensland, Brisbane, QLD 4072, Australia; 'Faculty of Paediatrics, Makerere University College of Health Sciences, P.O. Box 7072, Kampala, Uganda; ${ }^{m}$ Medical Research Council, Clinical Trials Unit at University College London, London, WC2B 6NH, UK;

${ }^{n}$ Department of Global Health and Development, London School of Hygiene \& Tropical Medicine, London, WC1E 7HT, UK.
\end{abstract}

*Corresponding author: Present address: Joint Clinical Research Centre, P.O. Box 10005, Kampala, Uganda; Tel: +256772587449; E-mail: gabongomera@jcrc.org.ug

Received 18 August 2016; revised 25 December 2016; editorial decision 5 February 2017; accepted 22 February 2017

Background: We conducted unlinked cross-sectional population-based surveys in Northern Uganda before and after antiretroviral therapy (ART) provision (including Option B+ [lifelong ART for pregnant/breast-feeding women]) at a local primary care facility (Lira Kato Health Centre [HC]). Prior to decentralisation, people travelled 56-76 km round-trip for ART; we aimed to evaluate changes in uptake of HIV-testing, ART coverage and access to ART following decentralisation.

Methods: A total of 2124 adults in 1351 households in two parishes closest to Lira Kato HC were interviewed using questionnaires between March and April 2013 and 2123 adults in 1229 households between January and March 2015.

Results: Adults reporting HIV-testing in the last year increased from 1077/2124 (50.7\%) to 1298/2123 $(61.1 \%)$ between surveys $(p<0.001)$. ART coverage increased from $74 / 136(54.4 \%)$ self-reported HIV-positive adults in 2013 to 108/133 (81.2\%) in 2015 ( $p<0.001)$. Post-decentralisation, 47/108 (43.5\%) of those on ART were in care at Lira Kato HC (including 37 new initiations). Most of the remainder (47/61, 77\%) started ART prior to any ART provision at Lira Kato HC; the most common reason given for not accessing ART locally was concern about drug-stock-outs (30/59, 51\%).

Conclusions: HIV-testing and ART coverage increased after decentralisation combined with Option B+ roll-out. However, patients on ART before decentralisation were reluctant to transfer to their local facility.

Keywords: Antiretroviral therapy decentralisation, HIV services, Population survey, Sub-Saharan Africa, Uganda

\section{Introduction}

Decentralisation of HIV care to get treatment close to where people live is essential to achieve the UNAIDS target of ensuring $90 \%$ of HIV positive individuals know their status, $90 \%$ of diagnosed HIV positive individuals receive sustained antiretroviral therapy (ART) and $90 \%$ of people on ART have suppressed viral load (90-90-90) by 2020. ${ }^{1}$ The recent WHO guidelines recommend 


\section{G. Abongomera et al.}

all individuals diagnosed with HIV should be initiated on ART at any CD4 cell count, further emphasizing that lack of access to CD4 cell count testing should not be a barrier to starting treatment and thus simplifying the ART initiation process in remote rural areas. $^{2}$

Uganda has an estimated adult HIV prevalence of $7.3 \%{ }^{3}$ According to the Uganda Ministry of Health $(\mathrm{MOH})$ semi-annual ART report (January-June 2014), 1211768 adults and 193500 children are currently living with HIV, of whom, 629212 (51.9\%) adults and 51302 (26.5\%) children are on ART. ${ }^{4}$ If Ugandan guidelines follow the WHO recommendation of treatment for all, the number of people in need of ART will further increase, reinforcing the need to decentralise care to all health facilities. Although the estimated adult HIV prevalence in rural areas is slightly lower than in urban areas (6.9\% versus $8.4 \%$ ), $82 \%$ of the population live in rural areas, ${ }^{3,5}$ highlighting the importance of scaling up decentralised HIV treatment and care services.

Studies evaluating decentralisation of HIV care and ART services to primary level facilities generally show this is a successful strategy for expanding HIV care, highlighting lower loss to follow-up (LTFU), better adherence and improved retention in care in comparison to secondary health facilities. ${ }^{6-11}$ Most information on ART scale-up focuses on adults, although lower mortality and LTFU have also been reported with successful ART rollout to children. ${ }^{12,13}$ Decentralisation has been shown to be beneficial especially to poorer individuals and those living in rural areas who are less likely to access HIV testing and treatment services due to the distance to facilities and transport costs. $^{14-16}$

The Lablite project investigated strategies for cost-effective and safe roll out of HIV treatment to primary health facilities in Uganda, Malawi and Zimbabwe in collaboration with respective ministries of health. ${ }^{17}$ In Uganda, we conducted a population survey immediately prior to decentralisation of ART to the local primary health care facility, Lira Kato Health Centre $(\mathrm{HC})$, in Lapono sub-county in the Agago district of Northern Uganda, a remote poor rural area, with low levels of education where most individuals rely on farming for their livelihoods (Supplementary Tables 1 and 2). ${ }^{18}$ HIV services available at Lira Kato $\mathrm{HC}$ before decentralisation of ART were HIV-testing and drugs for elimination of mother to child transmission of HIV (EMTCT). HIV positive individuals in Lapono sub-county were only able to access ART by travelling long distances to the district hospital (Kalongo Hospital) or to Patongo Health Centre (56 or $76 \mathrm{~km}$ round trip respectively), the majority on foot; despite this, $54 \%$ of adults who self-reported being HIV positive were receiving ART, although few children were accessing testing and treatment. ${ }^{18}$

ART for Option B+ (lifelong ART for all pregnant/breastfeeding women) was provided at Lira Kato HC from April 2013 and general ART for all HIV positive individuals eligible for treatment from September 2013. In this paper, we report results of a repeat population survey in the same area approximately 20 months after Option B+ roll out and 15 months after general ART provision at Lira Kato HC. Objectives were to describe the knowledge of local ART provision and to compare results with the survey pre-decentralisation with respect to: uptake of HIV testing in the last year; coverage of ART; and access to ART, including choice of health care facility.

\section{Methods}

\section{Study setting}

We conducted two unlinked cross-sectional population-based surveys in Lapono sub-county in Agago district, before and after decentralisation of ART services to Lira Kato HC. The first survey was conducted between February and April 2013 (Population Based Survey 1; PBS1).The repeat survey was conducted between January and March 2015 (Population Based Survey 2; PBS2).

According to the health facility structure in Uganda, Lira Kato $\mathrm{HC}$ is categorized as a Health Centre III (HC III); it is headed by a clinical officer, serves a sub-county and provides inpatient care, outpatient, antenatal, immunization and outreach services and environmental health. Patongo $\mathrm{HC}$ is categorized as a Health Centre IV (HC IV), is headed by a medical officer, serves a sub-district and additionally provides surgery, supervision of the lower HCs, data collection and health service planning. Kalongo Hospital is the district hospital and is headed by a senior medical officer, serves the district and additionally provides training and supervision of staff at the lower $\mathrm{HCs}$ and is the main referral facility for the district. All facilities are open seven days a week. Both Kalongo Hospital and Patongo HC have provided ART since 2006; Lira Kato HC referred patients to Kalongo Hospital for ART pre-decentralisation. Individuals within the catchment for Lira Kato $\mathrm{HC}$ are at most $9 \mathrm{~km}$ and mostly less than $5 \mathrm{~km}$ from the $\mathrm{HC}$, whereas average distances from the sub-county to Kalongo Hospital and Patongo $\mathrm{HC}$ are $28 \mathrm{~km}$ and $38 \mathrm{~km}$ respectively.

Both surveys were carried out in two of the six parishes including approximately $20 \%$ of villages in Lapono sub-county (Figure 1). All villages in the two parishes closest to Lira Kato $\mathrm{HC}$ were included. One village was used for the pilot (excluded from both main surveys) and 17 villages were included in the main surveys. Every household in each village was mapped and approached during the surveys. Community meetings were held in collaboration with local leaders to encourage participation of the villagers in the survey. Prior to PBS1, village mapping was done using geographical positioning system (GPS) technology for 14 villages and manual mapping for three villages, with additional guidance from a village member to locate dwellings and demarcate village boundaries within the study area. Manual mapping to update the previous maps from PBS1 was done prior to PBS2.

\section{Data collection}

Both surveys were carried out in the same way. Two field supervisors led a survey team of four members trained in data collection and fluent in Luo (the local language in Agago District). A local village member guided an interviewer to each household but did not remain for the interviews. The interviewer returned later if household members were not at home; up to two visits were made to each household. Questionnaires were used to collect socioeconomic data and information on children from the household head (PBS1 and PBS2 Household questionnaires, Supplementary Information) and individual demographic and health-related information from up to three adults aged 15-59 years in each household (PBS1 and PBS2 


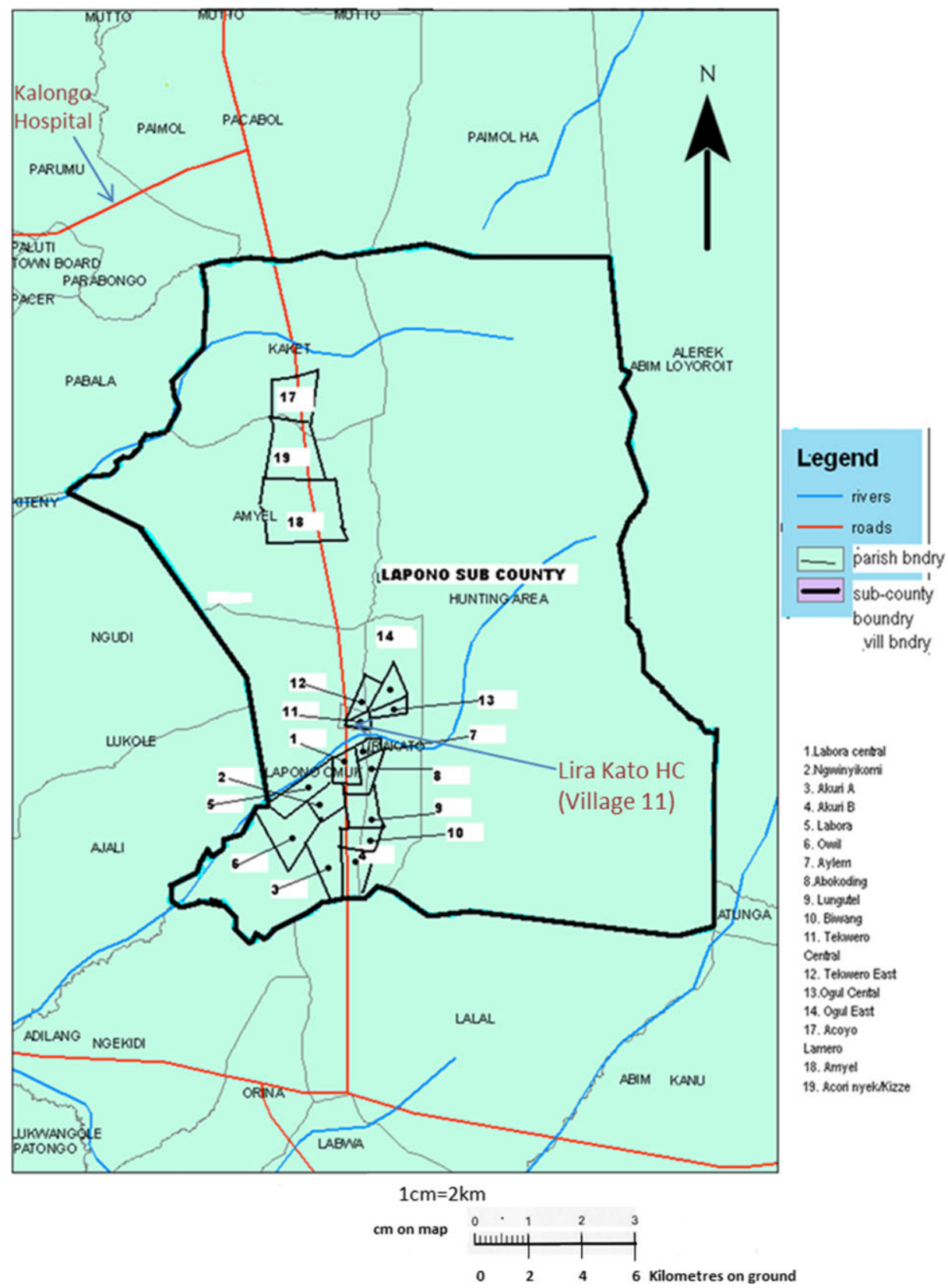

Figure 1. Map of Lapono sub-county. Patongo Health Centre is off the map to the bottom left-hand corner.

Individual questionnaires, Supplementary information) (in a small number of households more than three adults were interviewed [Table 1]; all data were included in the analysis). Individual health-related information on HIV-testing, HIVstatus and, for HIV-positive individuals, information on HIV care and treatment was included. Minor amendments were made to the questionnaires prior to PBS2, to capture knowledge of availability of ART at Lira Kato HC and reasons for not accessing ART at Lira Kato HC, where applicable. Interviews were held with 2124 adults from 1351 households in PBS1 and 2123 adults from 1229 households in PBS2 (Table 1). Respondents were not linked between surveys but household identifier numbers were maintained; 747/1401 (53.3\%) of households with a completed household questionnaire in PBS1 completed the household questionnaire in PBS2.

\section{Statistical analysis}

We used descriptive statistics for most analyses. In line with study objectives we evaluated differences between pre- and post-decentralisation surveys in self-reported HIV testing in the last year, EMTCT coverage and uptake of ART. We also investigated any changes in the proportions attending Lira Kato $\mathrm{HC}$ for HIV testing and for antenatal care (ANC). Villages 17, 18 and 19 were grouped as the villages furthest from Lira Kato $\mathrm{HC}$ and 


\section{G. Abongomera et al.}

Table 1. Summary of households approached during the surveys

\begin{tabular}{lcc} 
Number of households & PBS1 & PBS2 \\
\hline Total households mapped & 1895 & 1964 \\
Household questionnaire completed & $1401(73.9 \%)$ & $1316(67.0 \%)$ \\
$\geq 1$ adult individual questionnaire completed & $1351(71.3 \%)$ & $1229(62.6 \%)$ \\
1 adult interviewed & 753 & 558 \\
2 adults interviewed & 454 & 494 \\
3 adults interviewed & 116 & 138 \\
$>$ adults interviewed $^{\text {a }}$ & 28 & 39
\end{tabular}

PBS1: Population-based Survey 1 (before decentralisation); PBS2: Population-based Survey 2 (after decentralisation).

a The protocol allowed to interview up to three adults per household, although all interviews were included in analysis.

closest to Kalongo hospital (Figure 1); we describe ART uptake in these villages versus the remainder. Differences were tested using random effects logit models including a random effect for village. All data were analysed using Stata 12.1 (StataCorp LP, College Station, TX, USA).

\section{Ethics}

Approval for the study protocol was obtained from the Joint Clinical Research Centre/Research Ethical Committee (JCRC/ REC) and from the Uganda National Council for Science and Technology (UNCST) and Office of the President of the Republic of Uganda. All study participants provided written informed consent. The interviews were conducted within the household of the participants with one participant being interviewed at a time. Interviewers sought a private space for each interview in the home or surrounding compound.

\section{Results}

\section{Study participants}

In PBS2, 2123 individuals were interviewed including 821 (38.7\%) males and 1302 (61.3\%) females; numbers were similar in PBS1 (755/2124, 35.5\% males and 1369/2124, 64.5\% females). Household socio-economic indicators and participants' individual characteristics were similar in both surveys (Supplementary Tables 1 and 2).

Table 2. Self-reported HIV testing and self-reported HIV prevalence

\begin{tabular}{|c|c|c|c|c|c|c|c|}
\hline & $\begin{array}{l}\text { PBS1 } \\
n=755\end{array}$ & $\begin{array}{l}\text { PBS2 } \\
n=821\end{array}$ & $\begin{array}{l}\text { PBS1 } \\
n=1369\end{array}$ & $\begin{array}{l}\text { PBS2 } \\
n=1302\end{array}$ & $\begin{array}{l}\text { PBS1 } \\
n=2124\end{array}$ & $\begin{array}{l}\text { PBS2 } \\
n=2123\end{array}$ & $\begin{array}{l}\text { Test for } \\
\text { difference }(p)\end{array}$ \\
\hline \multicolumn{8}{|l|}{ HIV testing in the last year } \\
\hline Tested $\geq$ once in the last year & $406(53.8 \%)$ & $584(71.1 \%)$ & $671(49.0 \%)$ & $714(54.8 \%)$ & 1077 (50.7\%) & 1298 (61.1\%) & $<0.001^{\mathrm{c}}$ \\
\hline \multicolumn{8}{|l|}{ Most recent HIV test facility ${ }^{a}$} \\
\hline Kalongo Hospital & $119(29.3 \%)$ & $124(21.2 \%)$ & $83(12.4 \%)$ & $80(11.2 \%)$ & $202(18.8 \%)$ & $204(15.7 \%)$ & \\
\hline Other facilities & 137 (33.7\%) & $109(18.7 \%)$ & $381(56.8 \%)$ & $289(40.5 \%)$ & $518(48.1 \%)$ & $398(30.7 \%)$ & \\
\hline HIV prevalence ${ }^{a}$ & $5(1.2 \%)$ & $9(1.5 \%)$ & $31(4.6 \%)$ & $12(1.7 \%)$ & $36(3.3 \%)$ & $21(1.6 \%)$ & $0.007^{c}$ \\
\hline \multicolumn{8}{|l|}{ HIV testing ever } \\
\hline Ever tested & $574(76.0 \%)$ & $734(89.4 \%)$ & $1156(84.4 \%)$ & $1173(90.1 \%)$ & 1730 (81.5\%) & 1907 (89.8\%) & $<0.001^{\mathrm{c}}$ \\
\hline HIV prevalence ${ }^{b}$ & $34(5.9 \%)$ & $39(5.3 \%)$ & $102(8.8 \%)$ & $94(8.0 \%)$ & $136(7.9 \%)$ & $133(7.0 \%)$ & $0.30^{c}$ \\
\hline
\end{tabular}




\section{Knowledge of ART provision at nearest health facility in PBS2}

After introduction of ART provision at Lira Kato HC, 1454/2107 (69.0\%) participants reported Lira Kato HC as the nearest health facility with ART provision; 618/2107 (29.3\%) still thought Kalongo hospital was the nearest ART facility. Knowledge of ART provision at Lira Kato HC depended on village location; 324/771 $(42.0 \%)$ participants in the three villages furthest from Lira Kato $\mathrm{HC}$ reported ART was available there (with most reporting Kalongo Hospital as the nearest facility with ART) compared with $1130 / 1336$ (84.6\%) in the 13 villages closer to the HC. Knowledge of ART provision at Lira Kato HC did not vary by age, sex or education (data not shown), and was mostly acquired from friends/relatives (640/1452 [44.1\%]) or through attendance at Lira Kato HC for other services (432/1452 [29.8\%]), while $148 / 1452(10.2 \%)$ heard about it through community talks ( $n=2$ missing data).

\section{HIV testing in last $\mathbf{1 2}$ months and HIV prevalence}

The proportion of people who reported testing for HIV at least once in the last year had increased from $1077 / 2124(50.7 \%)$ in 2013 to $1298 / 2123(61.1 \%)$ in 2015 ( $p<0.001)$, with increases seen in men and women (Table 2, Figure 2). Among those who tested in the last year, most recent HIV tests were more likely to be at Lira Kato HC in PBS2 than in PBS1; HIV tests at Lira Kato $\mathrm{HC}$ in the past year increased nearly twofold between surveys from 357/2124 (16.8\%) in PBS1 to 696/2123 (32.8\%) participants in PBS2 (Table 2, Figure 2). Self-reported HIV prevalence in individuals ever-tested was similar in both surveys $(136 / 1730$ [7.9\%] in PBS1; 133/1907 [7.0\%] in PBS2); prevalence was lower

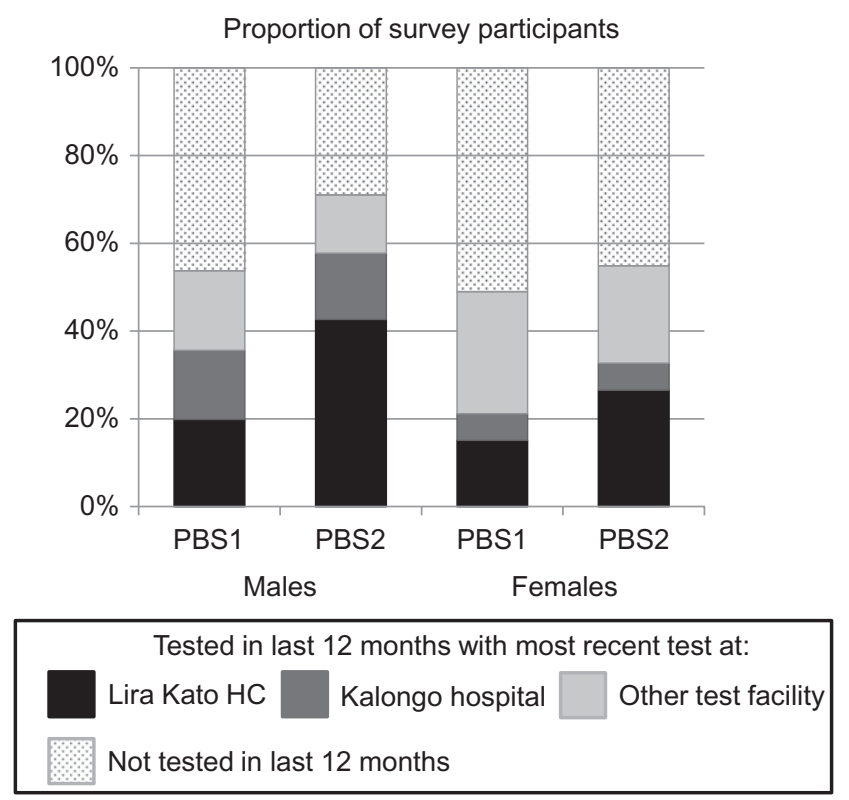

Figure 2. Self-reported HIV testing in the last 12 months and most recent test facility before and after antiretroviral therapy (ART) provision at the local primary care facility (Lira Kato Health Centre). PBS1: Population-based Survey 1 (before decentralisation); PBS2: Populationbased Survey 2 (after decentralisation). in individuals who had tested in the last year in PBS2 (21/1298 [1.6\%]) than in PBS1 (36/1077 [3.3\%]) $(p=0.007)$.

\section{ANC and EMTCT}

At the time of PBS1, rates of attendance at antenatal care during pregnancy and HIV-testing during pregnancy were both high. ${ }^{18}$ Similarly, in PBS2, 576/579 (99.5\%) women who had given birth in the two years preceding the survey attended antenatal care; $15 / 576$ (2.6\%) were already diagnosed with HIV (including eight on ART). Of the remainder, 558/561 (99.5\%) reported testing for HIV and 13/561 (2.3\%) tested positive; 14/ 15 (93\%) women diagnosed pre-pregnancy and 12/13 (92\%) women newly diagnosed in antenatal care reported receiving ART for themselves to prevent transmission to their baby. Although not statistically significant, ART coverage in newly diagnosed women was higher than in PBS1 (12/13 [92\%] vs 15/ 23 [65\%]; $p=0.12$ ). HIV-positive women who gave birth in the two years prior to survey were marginally more likely to attend Lira Kato HC for antenatal care in PBS2 (19/28 [68\%]) than in PBS1 (12/27 [44\%]) $(p=0.10)$.

\section{HIV care and treatment in adults}

Most HIV-positive adults with self-reported HIV diagnosis (including those diagnosed through antenatal care) were in HIVcare in both surveys (124/136 [91.2\%] in PBS1; 125/133 [94.0\%] in PBS2). At the time of PBS1, 74/136 (54.4\%) individuals were on ART compared with 108/133 $(81.2 \%)$ in PBS2 $(p<0.001)$. The proportion of men on ART increased from 20/34 (59\%) to $27 / 39$ $(69 \%)(p=0.17)$ and women on ART increased from 54/102 $(52.9 \%)$ to $81 / 94(86 \%) \quad(p<0.001)$. Very few individuals had taken ART in the past but were not currently on ART (seven in PBS1, one in PBS2). Sixteen of 81 (20\%) of women on ART at the time of PBS2 initiated ART post Option B+ roll-out either during pregnancy or within one year of giving birth; without Option B+ (assuming none of these women would have otherwise started ART), ART coverage would have reached 92/133 (69.2\%) (compared to 74/136 [54.4\%] in PBS1, $p=0.003)$.

During PBS1, 69/74 (93\%) adults on ART received care either at Kalongo Hospital or Patongo $\mathrm{HC}$ (average $56 \mathrm{~km}$ and $76 \mathrm{~km}$ round trip from Lapono sub-county respectively); the remainder were being seen elsewhere (three people) or the information was missing (two people). ${ }^{18} \mathrm{HIV}$-positive individuals living in the three villages closest to Kalongo Hospital (villages 17, 18, 19 [Figure 1]) were more likely to be on ART than those living further away (44/63 [70\%] vs 30/73 [41\%]; $p=0.001)$. Postdecentralisation, $47 / 108$ (43.5\%) of those on ART were in care at Lira Kato HC, 58/108 (53.7\%) were in care either at Kalongo Hospital $(n=55)$ or Patongo $\mathrm{HC}(n=3)$ and the remaining 3/108 $(2.8 \%)$ were at other facilities (Table 3, Figure 3). In PBS2, proportions of HIV-positive individuals on ART were similar in the three villages closest to Kalongo Hospital and the remainder (33/41 [80\%] vs 75/92 [82\%]; $p=0.89)$. All individuals receiving ART at Lira Kato HC went there on foot. The median selfreported time taken for an individual to travel to Lira Kato $\mathrm{HC}$, receive ART and return home was $5 \mathrm{~h}(3-6 \mathrm{~h})$; this was 


\section{G. Abongomera et al.}

Table 3. Current HIV care facility by time of antiretroviral therapy (ART) initiation in adults in PBS2 (Population-based Survey 2 [after decentralisation])

Time of ART initiation

HIV care facility at time of interview

\begin{tabular}{|c|c|c|c|c|c|c|}
\hline & \multicolumn{2}{|c|}{$\begin{array}{l}\text { Kalongo Hospital or Patongo } \\
\text { Health Centre }\end{array}$} & \multicolumn{2}{|c|}{ Lira Kato Health Centre } & \multicolumn{2}{|l|}{ Other } \\
\hline & Initiating facility & Transfer-in & Initiating facility & Transfer-in & Initiating facility & Transfer-in \\
\hline Before April $2013^{a}$ & 44 & 2 & 0 & 5 & 1 & 0 \\
\hline April 2013 to August $2013^{b}$ & 3 & 0 & 4 & 2 & 0 & 0 \\
\hline September $2013^{c}$ to February 2014 & 3 & 0 & 12 & 0 & 1 & 0 \\
\hline March 2014 to August 2014 & 4 & 1 & 8 & 2 & 0 & 0 \\
\hline September 2014 to interview ${ }^{d}$ & 1 & 0 & 13 & 1 & 1 & 0 \\
\hline Total & 55 & 3 & 37 & 10 & 3 & 0 \\
\hline
\end{tabular}

a No ART was available at Lira Kato Health Centre prior to April 2013.

${ }^{b}$ Option B+ was available at Lira Kato Health Centre.

c General ART was available at Lira Kao Health Centre from September 2013.

d Interview was conducted between January and March 2015.

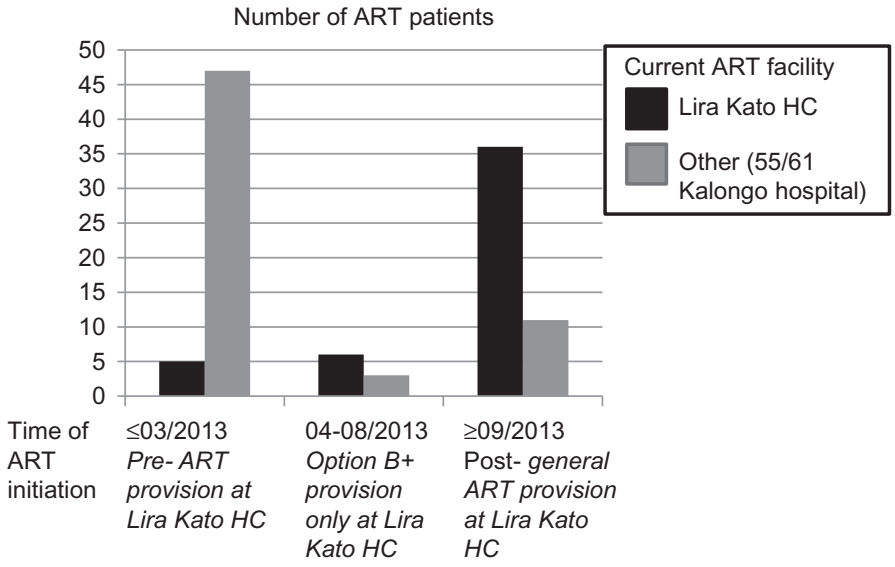

Figure 3. Current antiretroviral therapy (ART) facility at time of interview (Population-based Survey 2 [PBS2]) after decentralisation by time of ART initiation.

substantially less than time taken to access ART across all facilities in PBS1 (12 h [10-24 h]).

When interviewed for PBS2, only 5/52 (10\%) individuals who started ART prior to April 2013 were receiving ART at Lira Kato $\mathrm{HC}$, having transferred there post ART initiation, whereas 6/9 (67\%) individuals who started ART between April and August 2013 (including four initiations at Lira Kato [all for Option B+] and two transfers) and 36/47 (77\%) individuals who started ART from September 2013 onwards (33 initiations, three transfers) were receiving ART at Lira Kato $\mathrm{HC}$ (Table 3, Figure 3).

In total, only 10/71 (seven from Kalongo Hospital, two from Patongo $\mathrm{HC}$ and one from another facility) patients who started ART elsewhere had transferred to Lira Kato HC for ART (Table 3). The primary reasons given by 59/61 individuals for not accessing ART at Lira Kato $\mathrm{HC}$ included concerns about drug stock-outs
(30/59 [51\%]), starting ART before ART availability at Lira Kato HC (13/59 [22\%]), lack of trust in clinical staff at Lira Kato HC (5/ 59 [8\%]), stigma (4/59 [7\%]) and others reasons (7/59 [12\%]); all 61 knew Lira Kato HC provided ART.

Of the 37 individuals who initiated ART at Lira Kato HC, 10 (27\%) were men, 14 (38\%) were women initiating for Option B+ and 13 (35\%) were non-B+ women. Excluding Option B+ women, $11 / 23$ (48\%) initiations were in individuals who tested HIV-positive prior to any ART provision at Lira Kato HC.

During PBS2, 3/47 individuals on ART at Lira Kato $\mathrm{HC}$ reported not being able to collect both ART and cotrimoxazole in the past year (one individual reported 2 occasions); reasons given were drugs out of stock $(n=2)$ and no healthcare worker available $(n=1)$. One of 55 individuals on ART at Kalongo Hospital had not been able to collect either drug because the clinic was closed; there were no other difficulties reported with drug collection at the clinic at Kalongo Hospital or the other secondary health facilities (test for difference [Lira Kato HC vs other facilities], $\mathrm{p}=0.20$ ). In contrast, only $1 / 47$ ART patients at Lira Kato HC had missed a clinic visit due to distance/cost vs 9/61 of those still attending more distant secondary facilities (test for difference [Lira Kato HC vs other facilities], $p=0.05$ ).

\section{HIV care and treatment in children}

In each survey, information for only eight HIV-positive children was provided; based on household numbers, we estimate two children were reported in both surveys. All HIV-positive children in PBS2 were in care and receiving both cotrimoxazole and ART in contrast to PBS1 in which $6 / 8$ children were on cotrimoxazole, and only 3/8 were on ART and 1/8 child had dropped out of care due to transport costs to Kalongo Hospital (Table 4). The majority of children attended Kalongo Hospital (5/7 in PBS1 and 5/8 in PBS2). Post-decentralisation, 3/8 children on ART were in care 
Table 4. Summary of the children with HIV children reported in both surveys

\begin{tabular}{|c|c|c|}
\hline Surveys & $\begin{array}{l}\text { PBS1 } \\
n=8\end{array}$ & $\begin{array}{l}\text { PBS2 } \\
n=8\end{array}$ \\
\hline \multicolumn{3}{|l|}{ Current age } \\
\hline$<2$ & 0 & 0 \\
\hline $2-5$ & 4 & 3 \\
\hline$>5$ & 4 & 5 \\
\hline \multicolumn{3}{|l|}{ Current health facility } \\
\hline Lira Kato Health Centre & 2 & 3 \\
\hline Kalongo Hospital & 5 & 5 \\
\hline Not in care & 1 & 0 \\
\hline Currently on cotrimoxazole & 6 & 8 \\
\hline Currently on ART & 3 & 8 \\
\hline \multicolumn{3}{|l|}{ Transport to health facility } \\
\hline Own bike & 1 & 3 \\
\hline Borrowed bike & 1 & 2 \\
\hline Foot & 5 & 3 \\
\hline \multicolumn{3}{|l|}{ Primary carer } \\
\hline Mother & 5 & 6 \\
\hline Father & 1 & 1 \\
\hline Grandmother & 1 & 0 \\
\hline Siblings & 1 & 0 \\
\hline Uncle & 0 & 1 \\
\hline
\end{tabular}

ART: antiretroviral therapy; PBS1: Population-based Survey 1 (before decentralisation); PBS2: Population-based Survey 2 (after decentralisation).

locally at Lira Kato HC; one was a new initiation and two transferred to Lira Kato HC from another facility after starting ART. Reasons for not accessing ART locally included fear of drug stock-outs (2/5), not knowing ART was available at Lira Kato HC (1/5), awaiting transfer to Lira Kato HC (1/5) and reason unknown (1/5).

\section{Discussion}

Population-based surveys pre- and post-decentralisation of ART alongside Option $\mathrm{B}+$ roll-out at the local primary health care facility in Agago, Northern Uganda demonstrated benefits of decentralisation including an increase in the proportion of adults who reported testing for HIV in the last year, increased ART coverage and $44 \%$ of adults on ART accessing HIV care locally. The increase in recent HIV testing was driven by additional testing at the primary health care facility, particularly in men, which suggests that the availability of ART locally may have been a catalyst for the population seeking HIV testing; the safe male circumcision programme has run in the district for four years, covered both survey periods, and is likely to have contributed to the high levels of testing in men noted in both surveys. We did not collect information on reasons for recent HIV-testing which would have aided the understanding of these results. Encouragingly, although overall HIV prevalence was similar, among those tested in the last year, the proportion of selfreported positive tests declined between surveys. There was a significant increase in overall ART coverage, with a likely increase in EMTCT coverage; although undoubtedly, these increases were partly due to a change in HIV treatment guidelines which raised the CD4 threshold for ART initiation from 350 to 500 cell count, as well as country-wide roll-out of the Option B+ programme. ${ }^{19,20}$ Post-decentralisation new ART initiations were predominantly at the primary care facility, suggesting over time a higher proportion of ART patients will be treated locally. Around half of non-option $\mathrm{B}+$ initiations at the primary care facility were in patients who had tested positive before any ART provision was available there hence some individuals may have been eligible for treatment previously but unable to receive it.

The majority of patients who had started ART in secondary care prior to decentralisation of ART locally were reluctant to transfer to the primary care facility, despite the long distances they were travelling for treatment. A study conducted in Tanzania found that some patients preferred to attend more established health facilities rather than seeking care at the closest HIV care facilities. ${ }^{21}$ A study on patient attitudes towards decentralisation at an urban clinic in South Africa, showed some patients had reservations about receiving ART close to where they live due to concerns about stigma, lack of confidentiality, untrained staff and poor relations with nurses. ${ }^{22}$ However, in this study, fear of drug stock-outs was given by around half of the HIV-positive individuals as the reason for not transferring to the primary care facility for follow-up. Most of the remainder cited already starting ART elsewhere; it was unclear whether these individuals were unwilling to move, had not considered transferring, or were encouraged to remain at their current centre; all were aware of local availability of ART. Notably few individuals cited stigma and this did not appear to be a major concern among individuals on ART in this particular rural location. However, this study cannot address whether or not stigma remains a problem for individuals who had not been tested for HIV or did not reveal their HIV diagnosis during interview. Engaging staff at the secondary care facility (Kalongo Hospital) to encourage and promote transfer of individuals on ART from Lapono sub-county is one of the strategies being used to improve transfer of clients on ART to their local primary care facility. In addition community linkage facilitators and village health teams continue to emphasize the availability of comprehensive HIV care and treatment services locally during home visits; in the light of our results these activities may require strengthening.

A small number of patients reported problems collecting drugs due to stock-outs at the primary care facility but no issues were reported in secondary care facilities, reinforcing the need for stock management training for staff especially at primary level facilities to ensure timely ordering and correct projection of HIV commodities. However, patients were more likely to miss attending clinic at secondary facilities than at the primary care facility due to distance and cost.

The numbers of children in care still appeared low, even after decentralisation of ART to the local primary care facility. Given high EMTCT coverage, it may be that few new infant infections now occur. It is unclear to what extent older HIV-infected children are still outside HIV care; although guidelines include 


\section{G. Abongomera et al.}

provider-initiated counselling and testing, in practice this is often missed. ${ }^{23}$ Although numbers were small, the majority of the children in care attended Kalongo Hospital for ART, and some carers cited fear of drug stock-outs as the reason for not accessing ART locally; previous work has suggested that stockouts of paediatric ART may be more frequent than of adult ART. $^{24}$

Post-decentralisation, over two-thirds of the population reported the local primary care facility as the nearest facility where one could receive ART, although fewer participants were aware of local ART availability in the villages furthest from the health facility, suggesting community engagement could still be improved.

One of the limitations of the study was that we were unable to link individuals between surveys so our conclusions are limited to cross-sectional comparisons. Given there was substantial overlap between participating households, and must have been considerable overlap between individuals, we may have underestimated changes between surveys. Another limitation was that all information collected relied on self-report and we had no linked data from health facilities to verify participants' responses; in particular, we had no HIV-testing data thus we may have underestimated HIV prevalence. However, prior to the survey, the team of interviewers had residential training focusing on conducting community HIV surveys, including role play sessions on how to encourage accurate self-reporting of HIV status among respondents. There is evidence that response bias can be minimized by training interviewers to act ethically, build rapport and probe responses for more valid answers. ${ }^{25}$ Lastly, we included all households in the two parishes closest to the local health centre; whilst this means that we interviewed individuals most likely to benefit from decentralisation of ART services this could be a limitation of our findings if use of health services differs in populations living further away.

\section{Conclusion}

HIV-testing and ART coverage, increased after decentralisation combined with Option $\mathrm{B}+$ roll-out. Individuals starting ART mostly started locally. Patients who started ART prior to decentralisation were however reluctant to transfer to their local facility; the majority feared drug stock-outs. Although we identified only a low level of experience of stock-outs, the emphasis on stock management training for staff especially at primary level facilities to ensure timely ordering and correct projection for HIV commodities is critical. Patients attending secondary facilities were more likely to miss collecting ART due to distance/cost therefore reinforcing the need for decentralising ART services close to home.

\section{Supplementary data}

Supplementary data are available at International Health online (http://inthealth.oxfordjournals.org/).

Authors' contributions: GA contributed to the proposal writing; participated in interviews; conducted literature review and contributed towards interpretation and analysis of the results; writing manuscript. SK contributed towards the supervision of data entry; analysis and interpretation of the data; writing manuscript. DF contributed substantially towards the proposal writing; supervision of data entry; analysis and interpretation of the data; writing and editing the manuscript. JS and DMG contributed towards the study design; proposal writing; interpretation of the results; writing the manuscript. VM, AKC, LC and TM provided technical advice in proposal writing and editing the manuscript. PR, ANP, $\mathrm{CK}, \mathrm{EK}, \mathrm{JH}, \mathrm{RC}$ and $\mathrm{CG}$ contributed towards study design; interpretation of results and reviewing of the manuscript. All authors read and approved the final manuscript. GA, DMG and DF are guarantors of the paper.

Acknowledgements: We thank the Agago District Data collection team: E Lubowa, W Aciro, C Opira, B Atim, P Okumu who conducted the interviews and collected data in the field during the population survey.

Funding: This work was supported by the UK Department for International Development (DFID) for the benefit of developing countries [GB-1-202037]. The views expressed in this paper are not necessarily those of DFID.

Competing interests: None declared.

Ethical approval: Approval for the study protocol was obtained from the Joint Clinical Research Centre/Research Ethical Committee (JCRC/REC) and from the Uganda National Council for Science and Technology (UNCST) and Office of the President of the Republic of Uganda.

\section{References}

1 UNAIDS. 90-90-90 An Ambitious Treatment Target to Help End the AIDS Epidemic. Geneva: UNAIDS; 2014.

2 WHO. Guideline on When to Start Antiretroviral Therapy and on PreExposure Prophylaxis for HIV. Geneva: World Health Organization; 2015.

3 Ministry of Health Uganda. Uganda AIDS Indicator Survey 2011. Kampala: Ministry of Health Uganda; 2012.

4 Ministry of Health Uganda. Status of Antiretroviral Therapy Services in Uganda. Semi-Annual ART Report for January - June 2014. Kampala: Ministry of Health Uganda; 2014.

5 Uganda Bureau of Statistics. 2015 Statistical Abstract. Kampala: Uganda Bureau of Statistics; 2015.

6 Fatti G, Grimwood A, Bock P. Better antiretroviral therapy outcomes at primary healthcare facilities: an evaluation of three tiers of ART services in four South African provinces. PLoS One 2010;5:e12888.

7 Chan AK, Mateyu G, Jahn A et al. Outcome assessment of decentralization of antiretroviral therapy provision in a rural district of Malawi using an integrated primary care model. Trop Med Int Health 2010; 15(Suppl 1):90-7.

8 Reidy WJ, Sheriff M, Wang C et al. Decentralization of HIV care and treatment services in Central Province, Kenya. J Acquir Immune Defic Syndr 2014;67:e34-40.

9 Boyer S, Ebokob F, Camara M et al. Scaling up access to antiretroviral treatment for HIV infection: the impact of decentralization of healthcare delivery in Cameroon. AIDS 2010;24(Suppl 1):S5-15.

10 Massaquoi M, Zachariah R, Manzi M et al. Patient retention and attrition on antiretroviral treatment at district level in rural Malawi. Trans R Soc Trop Med Hyg 2009;103:594-600. 
11 McGuire M, Pinoges L, Kanapathipillai $R$ et al. Treatment initiation, program attrition and patient treatment outcomes associated with scale-up and decentralization of HIV care in rural Malawi. PLoS One 2012;7:e38044.

12 Fayorsey RN, Saito S, Carter RJ et al. Decentralization of pediatric HIV care and treatment in five sub-Saharan African countries. J Acquir Immune Defic Syndr 2013;62:e124-30.

13 van Dijk JH, Moss WJ, Hamangaba F et al. Scaling-up access to antiretroviral therapy for children: a cohort study evaluating care and treatment at mobile and hospital-affiliated HIV clinics in rural Zambia. PLoS One 2014;9:e104884.

14 Pinto AD, van Lettow $M$, Rachlis $B$ et al. Patient costs associated with accessing HIV/AIDS care in Malawi. J Int AIDS Soc 2013;16:18055.

15 Muula AS. Who accesses antiretroviral drugs within public sector in Malawi? Croat Med J 2006;47:356-9.

16 Makwiza I, Nyirenda L, Bongololo $G$ et al. Who has access to counseling and testing and anti-retroviral therapy in Malawi - an equity analysis. Int J Equity Health 2009;8:13.

17 MRC Clinical Trials Unit at UCL. Optimising clinical care strategies and laboratory monitoring for cost-effective roll-out of antiretroviral therapy in Africa: the Lablite project. http://www.ctu.mrc.ac.uk/our_research/ research_areas/hiv/studies/lablite/ [accessed 8 February 2017].

18 Abongomera G, Kiwuwa-Muyingo S, Revill P et al. Population level usage of health services, and HIV testing and care, prior to decentralization of antiretroviral therapy in Agago District in rural Northern Uganda BMC Health Serv Res 2015;15:527.

19 Ministry of Health Uganda. Addendum to the National Antiretroviral Treatment Guidelines. Kampala: Ministry of Health Uganda; 2013.

20 Ministry of Health Uganda. EMTCT Communication Plan to Support the Roll Out of Option B+ for EMTCT in Uganda. Kampala: Ministry of Health Uganda; 2012.

21 Ostermann J, Whetten K, Reddy E et al. Treatment retention and care transitions during and after the scale-up of HIV care and treatment in Northern Tanzania. AIDS Care 2014;26:1352-8.

22 Mukora R, Charalambous S, Dahab M et al. A study of patient attitudes towards decentralisation of HIV care in an urban clinic in South Africa. BMC Health Serv Res 2011;11:205.

23 Penazzato M, Revill P, Prendergast AJ et al. Early infant diagnosis of HIV infection in low-income and middle-income countries: does one size fit all? Lancet Infect Dis 2014;14:650-5.

24 Chan AK, Ford D, Namata $\mathrm{H}$ et al. The Lablite project: A crosssectional mapping survey of decentralized HIV service provision in Malawi, Uganda and Zimbabwe. BMC Health Serv Res 2014;14: 352.

25 Johnston LG, Sabin LM, Prybylski D et al. The importance of assessing self-reported HIV status in bio-behavioural surveys. Bull World Health Organ 2016;94:605-12. 